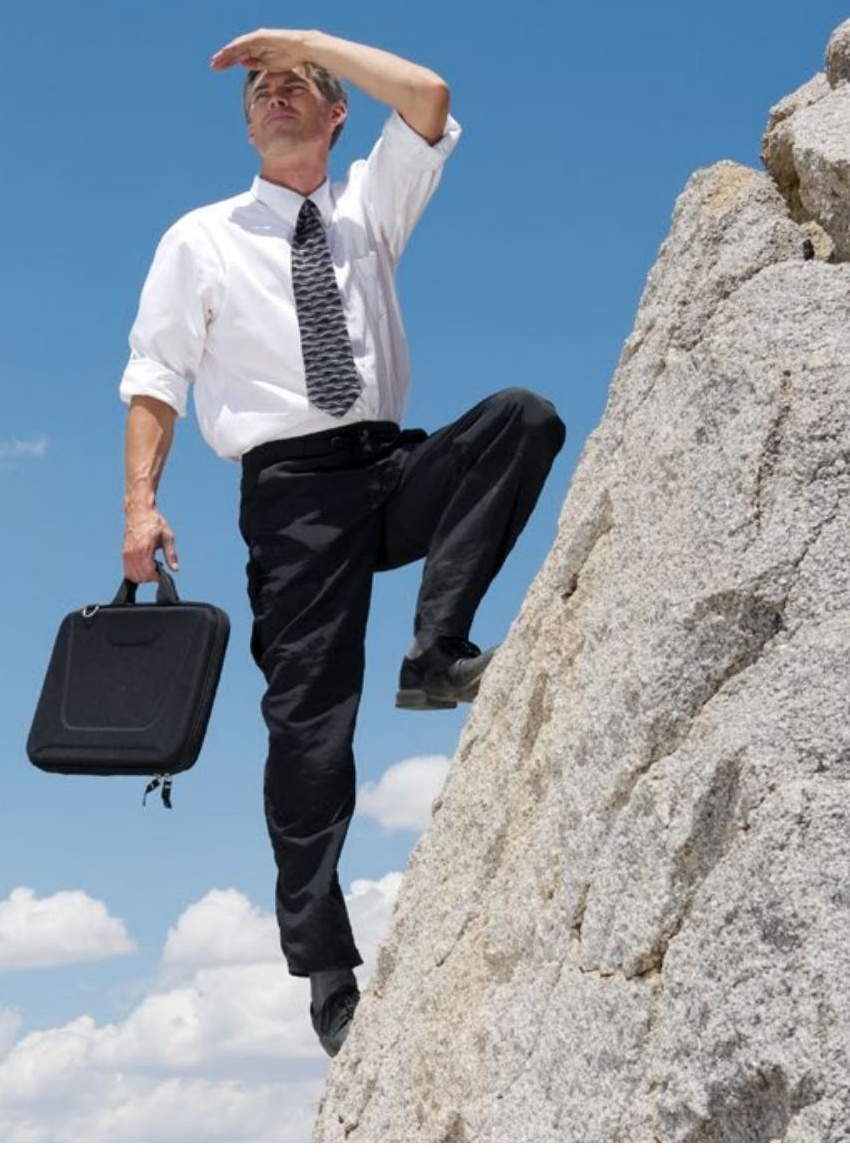

\title{
Das Gesundheitswesen steht vor großen Herausforderungen
}

normalerweise gehen einem Autor beim Schreiben von Vorworten zu Periodika irgendwann einmal die Themen aus. Ganz anders im deutschen Gesundheitswesen. Hier sorgen auf der einen Seite die kassenärztliche Bundesvereinigung und das Bundesgesundheitsministerium durchgehend für Schlagzeilen (jüngst die Drohung mit dem Staatskommissar!), auf der anderen Seite ist es leider auch im Bereich der für die Mitglieder der ADK wichtigen Gebührenordnung für Ärzte und der Bundesärztekammer alles andere als ruhig. Gemeinsam scheint beiden Bereichen ein ausgesprochener Mangel an strategischem Management sowie Effizienz und Transparenz insbesondere auf der Ärzteseite.

Es ist erschreckend, wenn erst jetzt seitens der Bundesärztekammer ein Institut für die GOÄ gegründet werden soll, welches längst überfällig ist. Dritte wie die private Krankenversicherung haben längst Unternehmensberatungen wie McKinsey in ihrem Sinne beauftragt, dafür zu sorgen, dass die maximale Erhöhung der Gebühren eine Obergrenze von $6 \%$ nicht übersteigt.

Rechnet man den Verlust der Kaufkraft von 1983 bis heute, so ergibt sich eine notwendige Erhöhung der Gebührenordnung für Ärzte zwischen 72 und $75 \%$ je nach Region - wohlgemerkt

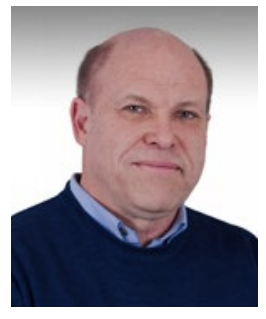

Dr. Matthias Herbst

Generalsekretär Arbeitsgemeinschaft

Ästhetische Dermatologie und

Kosmetologie e. V.

nur zum Ausgleich des Kaufkraftverlustes. Es stellt sich also die Frage: Ist die Politik bereit und die private Krankenversicherung in der Lage, eine derartige Erhöhung der Gebühren zu managen? Die Antwort ist einfach: Die Politik will es nicht und die PKV kann es nicht. Man schleppt das Thema weiter in den nächsten Bundestagswahlkampf. SPD und Grüne wollen mit dem Dauerthema „Bürgerversicherung“ und „Einheitsrente“ bzw. „Grundeinkommen“ punkten. Hinzu kommt sicher noch das Thema „Vermögenssteuer“, gerade durch die Linken und die 
Grünen. Rot-Rot-Grün steht im Bund vor der Tür. Die Schwarzen pulverisieren sich gerade!

Viel zu lange haben die Verantwortlichen auf Ärzteseite gezögert, eine bekömmliche Anpassung der GOÄ in einem regelmäßigen Zeittakt durchzusetzen und das Thema aus dem Verteilungskampf der Politik herauszuhalten. Die Politiker in Berlin haben es dagegen geschafft, ihr Honorar automatisch an die Entwicklung der Bruttolohnsumme in Deutschland anzupassen. Die Ärzte sind dazu offensichtlich nicht in der Lage. Überdies sorgen publizistische Feldzüge der Politik dafür, dass das Ansehen der Ärzte regelmäßig via Presse geschädigt wird. Ich erinnere nur an das Gesetzgebungsverfahren zum „AntiKorruptionsgesetz" sowie an die neueste Aktion des Bundesgesundheitsministeriums mit dem Motto: „Die Patienten verstehen die Ärzte nicht“.

Nunmehr muss man sich endlich von der gewohnten Ruhe im Gesundheitswesen verabschieden und die Ärzteschaft muss im Sinne der Patienten mobilmachen. Ohne Streikbereitschaft und weitere drastische, möglichst intelligente Maßnahmen seitens der Ärzte geht es nicht. Es ist Zeit, dass zumindest die regionalen Strukturen aufwachen und unseren Vertretern Dampf machen. Es muss allen klar sein, dass es nur gemeinsam - Hausarzt und Facharzt - eine auskömmliche Zukunft im Gesundheitswesen geben kann. Der politische Versuch, die Ärzteschaft in Haus- und Fachärzte sowie weitere Gruppen zu spalten, ist durchsichtig und hinterhältig und ganz im Sinne der politisch gewünschten fachärztlichen Industrialisierung des Gesundheitswesens.

Eine Neufassung der gültigen Gebührenordnung ist somit vorerst obsolet. Es geht vielmehr darum, zunächst eine lineare nachprüfbare Erhöhung der Gebührenordnung zu erreichen. Erst wenn diese eine gewisse Höhe umfasst, lassen sich in der Folge einzelne Kapitel der GOÄ in Ruhe nacheinander an den aktuellen Stand der aktuellen Medizin anpassen. Wir dürfen uns unter anderem nicht der Gefahr aussetzen, dass durch eine neue GOÄ auch noch unsere zum Teil überlebensnotwendigen Selbstzahlerleistungen dem politischen Rotstift zum Opfer fallen.

Wenn der Gesundheitsminister erkennt, dass die Mehrheit der Ärzte sicherlich nichts dagegen hat, wenn der Bundesrechnungshof jährlich die Ausgaben der KBV überprüft und die Vergütung der Funktionäre nach nachprüfbaren einheitlichen
Kriterien leistungsbezogen erfolgt, so bin ich sicher, dass wir auch in anderen Bereichen sinnvolle und tragbare Kompromisse erzielen werden. Umgekehrt muss aber auch klar sein, dass die Ärzteschaft nicht der Befehlsempfänger eines Bundesgesundheitsministers ist und seine Vorgaben einfach abnickt.

Insbesondere in den jetzigen unruhigen Zeiten, wo täglich Krankenhäuser und Praxen Opfer von professionellen Hackern werden können und jeder Einzelne dadurch womöglich in seiner körperlichen Integrität bedroht ist, müssen Datenschutz und -sicherheit über IT-Wunschvorstellungen und Industrieinteressen gestellt werden.

Ist das Vertrauen des Bürgers erst einmal durch einen Zusammenbruch der Gesundheits-IT-Infrastruktur nachhaltig geschädigt (was in einem dezentral organisierten Gesundheitswesen bei den Aktivitäten gewisser Hackerkreise tagtäglich passieren kann), nimmt auch die Ärzteschaft Schaden. Wir dürfen uns also nicht durch das Androhen von Strafzahlungen bei nicht Erreichen von politisch gesteckten Zielen bezüglich des Ausbaus der IT-Infrastruktur erschrecken lassen, sondern müssen standfest und nachprüfbar zu den Grundfesten unseres Berufsstandes stehen, zu dem der demokratische Eid und die Verschwiegenheit vornehmlich gehören, und müssen so auch nachvollziehbar bei problematischen Entwicklungen „Nein“ sagen können. Mehr denn je gilt: Der Arzt und sein Patient sitzen in einem Boot, der Politiker und die Industrie in einem anderen.

Daten müssen noch in der Arztpraxis anonymisiert werden, die Bestimmungen des Sozialgesetzbuchs gehören im Sinne des präventiven Datenschutzes diesbezüglich hinterfragt und überarbeitet. Für das Bundesgesundheitsministerium bedeutet dies erst einmal mit dem BSI und der Gematik GmbH entsprechend Vorarbeit zu leisten, bevor weitere konkrete Vereinbarungen bezüglich IT-Ausbau und -Vernetzung mit der Ärzteschaft getroffen werden können. In diesem Sinne sind auch ärztliche Telematik-Projekte unter Vorbehalt zu stellen.

\section{Mit freundlichem Gruß}

Ihr

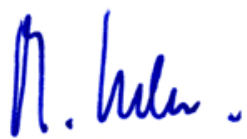

